\title{
SPRING Mission: Exploring the past and enabling the future of Mars
}

\section{Coordinating Authors}

John Roma (J.R.) Skok, SETI Institute

Suniti Karunatillake, Louisiana State University

Kris Zacny, Honeybee Robotics

Emily B. Hughes, Louisiana State University, Wesleyan University

Science Authors

Jennifer Blank, Blue Marble Space Institute of Science / NASA Ames

Research Center

Jessica Gaskin, NASA/MSFC

Amy Williams, University of Florida Kevin Cannon, University of Central Florida Jennifer Edmunson, NASA/MSFC Mario Parente, University of Massachusetts

A whitepaper to the NAS-NASA 2023 - 2032 Decadal Survey 


\section{Mission Overview}

In order to assess biosignatures and ancient signs of habitability, the Surface Probe Rover Investigating New Ground (SPRING) Mission will operate the Miniaturized Variable Pressure Scanning Electron Microscope (MVP-SEM) (Edmunson et al., 2016) on the martian surface. The MVP-SEM instrument includes mission configurations which address key NASA objectives. Here we focus on a single, specific mission profile to better develop the engineering and operational requirements: an astrobiological mission to a martian volcanic hot spring deposit, a target that will allow us to meet multiple high-level NASA science goals and leverage the expertise of the proposal team.

SPRING would feature compositional mapping capabilities via Energy Dispersive Spectroscopy (EDS). SPRING provides the necessary structure for MVP-SEM to map, sample and analyze the materials of Mars for astrobiology, geology, and engineering insight. MVP-SEM enables in-situ nanoimaging with a resolution of $50 \mathrm{~nm}$ across a field of view of $0.75 \mathrm{~mm}$. Nanoimaging with SEM is a fundamental laboratory technique to understand the physical and compositional properties of materials.

Nominally, SPRING would use a rover to deliver MVP-SEM close to the primary target site. To sample subsurface units, SPRING will deploy an impactor suite, the Mars Surface Probe (MSP), to excavate the target units at depth before the rover lands. Upon landing the rover, SPRING will explore the freshly excavated and associated surface targets. Sampling payload includes detachable Mars Mapping Drones (MMD) to map regional geology and retrieve small samples. The rover will feature cameras for geomorphic mapping, spectrometers for mineral mapping, a robotic arm for sampling and a sample processing station for MVP-SEM. Three science aims are supported: (1) determine if the martian spring deposits have evidence of physical biosignatures; (2) determine the environmental conditions of the ancient spring system and the volcanic history that drove it; and (3) characterize the locale for human exploration and in situ resource use (ISRU).

\section{Aims and Rationale}

Science Aims. SPRING would be driven by three main science aims that were motivated in part by goals outlined in the 2014 NASA Science Plan, Vision and Voyages decadal survey, the Committee on Astrobiology and Planetary Sciences and the Planetary Science Division Mid-Term report (NRC 2011; NASEM 2017).

\section{Aim 1: Detect the presence of physical biosignatures in the near surface of Mars}

SPRING will detect the presence of physical biosignatures (Figure 1) as physical cells or cellular organic matter. The extraordinary preservation of microbial cells in sinter deposits by entombment in a very fine matrix of silica particles is well established (Walter and Des Marais, 1993; Cady and Farmer, 1996; Walter, 1996; Renaut et al., 1998; Jones et al., 2000; Konhauser et al., 2001; Guido et al., 2010; Brasier et al., 2011; Campbell et al., 2015; Westall et al., 2015; McCollom et al., 2016; Munoz-Saez et al., 2016; Tarhan et al., 2016). Additional studies have focused on preserved organic matter in modern and relict (up to 900 year old) siliceous sinter deposits (Kaur et al., 2015, 2011, 2008; Pancost et al., 2005; Pancost et al., 2006; Gibson et al., 2014; Chaves Torres et al., 2019; Teece et al., submitted; Williams et al., submitted; Williams et al., 2020). The search for biosignatures motivates the entire Mars Sample Return (MSR) mission architecture, for which SPRING could work in tandem or independently. The SPRING payload could search samples for physical biosignatures before caching and return for confirmation, or search for biosignatures in landforms that would preferentially only have physical microbiosignatures, such as spring sinter sites. 
Aim 2: Characterize the local environmental and geological history that lead to habitability

If biosignatures are detected, it will be critical to understand the environment in which they formed; if biosignatures are not detected, it is important to understand the habitability and preservation potential of the site to contextualize the absence in detection. Specifically for spring deposits, environmental details, such as volumes, flow rates, temperature, $\mathrm{pH}$ of the ancient spring water, extent of geothermal activity, relative duration/fluctuation of sinter or geyser activity, and chemical variability of the source fluids must be quantified. Volcanic rocks will be characterized to determine the eruption history and composition to understand the hydrothermal systems' provenance and the volcanic history of Mars.
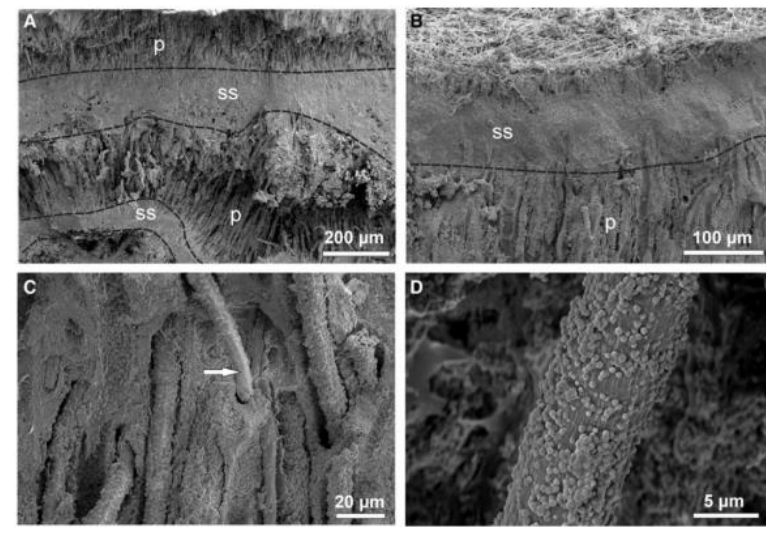

Figure 1. Scanning electron microscope images of palisade fabric within modern sinter from Orakei Korako, NZ; sample M1. (A) Alternating horizons of smooth silica (ss) and densely packed, vertically oriented, cyanobacterial sheaths ( $p$ ) that form the palisade sinter microfabric. (B) Uppermost horizon of filamentous fabrics (p), $\sim 100$ um below the sinter surface, immediately underneath a smooth silica (ss) horizon. (C) Palisade microfabric shows silicaencrusted, subvertically oriented cyanobacterial sheaths. Arrow indicates filament sheath not coated with opal-A spheres. (D) Individual cyanobacterial sheath partially encrusted with 0.5 m diameter opal-A silica spheres. (From Campbell et al., 2015)

\section{Aim 3: Characterize regional resources to prepare for human exploration}

The SPRING Mission will conduct mapping, compositional and morphological characterization of the regional resources for potential human safety and in-situ resource use (ISRU). Imaging and mapping campaigns would identify geologic units, while sample analysis would characterize the physical and compositional characteristics of the materials. That will allow understanding dust and particle hazards for health, compositional qualities for mineral and elemental extraction and physical and mechanical properties for ISRU.

\subsubsection{Science Rationale}

SPRING's payload and aims address many geology and astrobiology motivated NASA science goals and questions identified in the Decadal Survey and related documents (NRC 2011; NASEM 2017). For example, Table STM (Skok et al. 2020) maps the relationship between NASA science goals, SPRING mission objectives and mission instrument performance.

\subsubsection{Technology Aims}

We will use significant heritage on the landing system, rover platform, cameras, sensors and Mars Mapping Drone. SPRING will also add precision targeting for the Mars Surface Probe and main spacecraft, Mars helicopter mapping and sample collection capabilities, MVP-SEM instrument development, operations and sample handling. The multiple surface probes and drones create opportunities for networked operations, foreseeable for future space missions using multiple rover and human operators concurrently.

\subsubsection{Technology Rationale}

SPRING enables multiple NASA Technology needs outlined in the Visions and Voyages: A Midterm Review (NRC 2011; NASEM 2017). Table 4.1 of the report lists several current NASA Technology Roadmap Priorities for Mars exploration. Several of those would be directly addressed by SPRING including: (1) autonomous precision landing technology; (2) system autonomy (GNC, proximity operations, C\&DH, sampling operations, FIDH); (3) sample acquisition systems (ice 
penetration melt, drills; plume sampling; sample cache, delivery, and processing); (4) landing hazard avoidance systems; and (5) planetary protection techniques/material and component compatibility.

\subsection{Initial Mission Concept}

\subsubsection{Proposed Mission Target}

While SPRING would be designed initially for a Martian surface scenario, the core instrumentation and mission aims apply to other rocky and icy bodies of interest, including Mars, the Moon, asteroids, comets, and Europa. Accordingly, SPRING will reveal the technical, instrumental and financial trade space requirements to apply the concept to other target bodies.

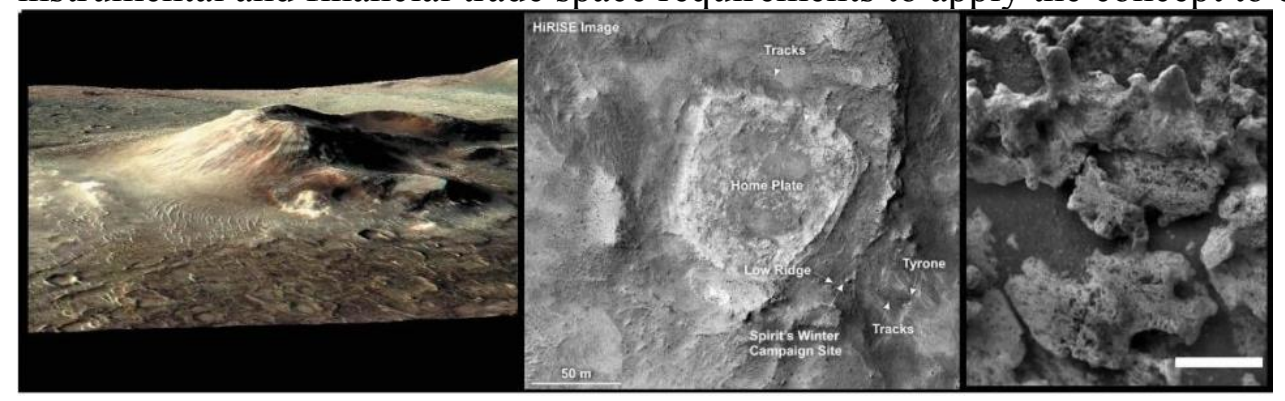

Figure 2. Silica sinter deposits on Mars. Left: Sinter deposits in Nili Patera. Mound is $\sim 500 \mathrm{~m}$ high. (Skok et al., 2010) Center: Sinter deposits in Columbia Hills. (Ruff et al., 2011) Right: Digitate structures and potential stromatolitic biosignatures from Columbia Hills. While bar is $10 \mathrm{~cm}$ across. (Ruff and Farmer, 2016)

On Mars, the SPRING Mission and MVP-SEM instrument payload would be well suited for several specific mission objectives and as a precursor to more advanced concepts. The mission would be perfectly aligned with the search for physical biosignatures in astrobiologically interesting locations. Hot Spring deposits like Home Plate in the Columbia Hills (Squyres et al., 2008) and the Nili Patera Caldera (Skok et al., 2010) could be mapped and examined for silica encased microfossils (Campbell et al., 2015) in a highly targeted search for biosignatures (cf. Figure 2). MVP-SEM would directly confirm such biosignatures with only in-situ samples. These locations would provide the targets to meet each of the science objectives that guide the development of the SPRING Mission.

1.3.2 SPRING Mission Spacecraft uses a Mars rover platform (Figure 3). The spacecraft systems will be divided into four main components that would work in tandem to accomplish the mission objectives. All four will launch and cruise together to Mars.

Cruise and Entry, Descent and Landing (EDL) Platform: The SPRING Mission Cruise and EDL Platform uses heritage equipment from Mars 2020, with the expectation that it will include any advances in targeting and precision landing technology and scaled to size of the SPRING Mission payload. The only addition would be docking ports on the Cruise stage for the Mars Surface Probe (MSP) attachments that will be deployed before atmospheric entry. The EDL Platform would contain cameras for the entry, descent and landing procedure and targeting and a spectrometer to measure the composition of the MSP material plume.

Mars Surface Probes (MSP) will be based on heritage from the Moon Impact Probe (MIP) (Goswami \& Annadurai, 2009) from the Indian Space Organisation (ISRO) and lessons learned from the Deep Space 2 mission (Smrekar et al., 1999). We will consider the tradeoffs of excavating biosignatures from depth, and the risk that the impact will disrupt the targeted samples. On approach the MSPs will fire toward the landing site. MSPs will impact the surface at $\sim 180 \mathrm{~m} / \mathrm{s}$, while transmitting data. The resulting impact would excavate up to 0.6 meters depth (Smreker et al., 1999), depending on the surface unit. Targeting part of the sinter field would expose material 
at depth that would have had little recent radiation or surface weathering exposure to degrade biosignatures (Dartnell et al., 2007).

SPRING Rover: The rover will accommodate the MVP-SEM, Mars Surface Cameras, Mars Mapping Spectrometer, Collection Arm and Sample Preparation Station. The power needs, source and payload capacity will determine the size and capacity of the rover and the required landing methods.

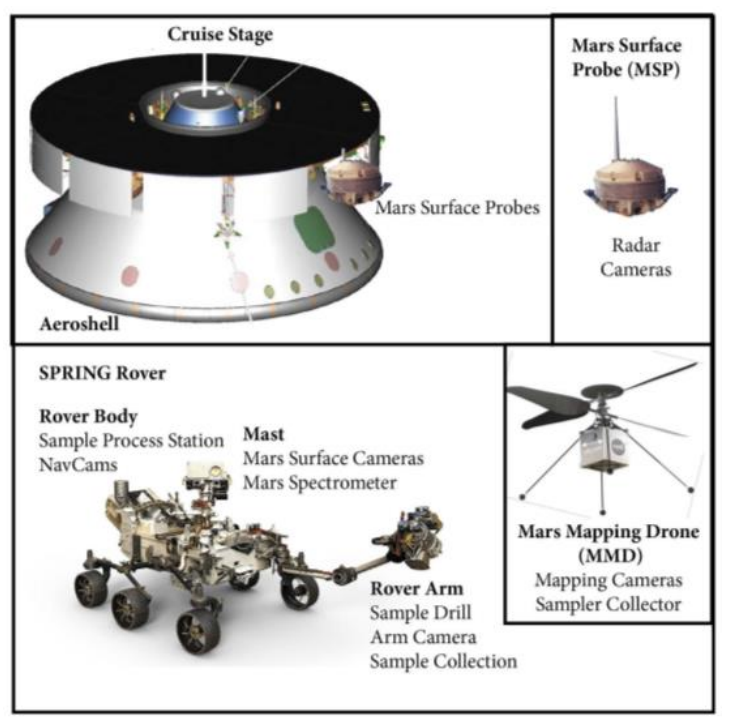

Figure 3: SPRING Mission spacecraft architecture. The cruise stage will carry and then release the MSP. The Aeroshell will deliver the Rover and MMD to the surface. Mars Mapping Drone (MMD): Onboard the SPRING Rover will be the detachable Mars Mapping Drones (MMD), emulating the JPL Mars Helicopter Scout (MHS) (Balaram et al., 2018). Those small vehicles will roam within range of the SPRING Rover base station to map surrounding terrain and retrieve samples for analysis on the Rover. MMD will allow for comprehensive mapping and possible collection of mission critical, but difficult to reach, samples.

\subsubsection{Science Payload}

Entry, Descent and Landing (EDL) Platform Payload will rely on Mars 2020 technology heritage. It will include instrumentation based on MSL's Entry, Descent and Landing Instrumentation 2 (MEDLI2) instrument suite that will enable Range Trigger and Terrain-Relative Navigation to target landing within $10 \mathrm{~km}$ or closer to the target (Wolf et al., 2011).

\section{Mars Surface Probe (MSP) Payload}

Radar Altimeter includes streaming data relays for probe tracking until impact. That would allow for data to use for fine targeting and to pre-arrival site characterization.

Video Imaging System includes streaming imaging on the probe for the first close range images of the landing site that can be used for precision targeting and later landing site adjustments.

\section{SPRING Rover Payload}

Miniaturized Variable Pressure Scanning Electron Microscope (MVP-SEM) is the primary instrument of SPRING. It has been developed through multiple NASA efforts (PIDDP, PICASSO) (Edmunson, et al., 2016) with proposing intended for MATISSE. It will have an imaging resolution of $50 \mathrm{~nm}$ for a field of view of $0.75 \mathrm{~mm}$. MVP-SEM will have compositional mapping capabilities with Energy Dispersive Spectroscopy (EDS) with a precision of finer than $\pm 5 \%$ for major elements and $\pm 25 \%$ for minor elements.

MVP-SEM will allow the Earth-based science team to answer questions related to the direct imaging and geochemical analysis of the martian surface. MVP-SEM will provide detailed information on the study of amorphous martian phases using a technique that does not rely on crystal structure, will provide imaging and calibrated EDS of phases present, will characterize potential biosignatures, and will assist in the identification of phases that may be useful for, or toxic to human explorers. MVP-SEM enables the identification of potential biosignatures through morphology, geochemical analysis, and element mapping. The instrument design allows modification for use on other planetary bodies such as the Moon (in high-vacuum mode, or if a 
gas is flown - in low-vacuum mode) and Earth. The MVP-SEM can also be used on orbiting platforms such as the International Space Station (ISS) and Lunar Gateway.

Mars Surface Cameras (MSC) are a high-resolution, multispectral stereo imager that would identify potential sampling sites in the field and evaluate them in the context of their surroundings. MSC would be used in studies of morphology, topography, and geology, as well as in studies of atmospheric dust and opacity. The selected cameras would be based on previous Mars rover heritage. Instrument will be paired with calibration target.

Mars Mapping Spectrometer (MMS) would use remote sensing spectroscopy to map the mineralogical composition of the surface for unit characterization and sample selection. Specific spectral ranges and bands will be considered as part of the instrument trade space. A Thermal Emission Spectrometer (e.g., Mini-TES) was used to identify silica at Home Plate, while the Nili Patera deposits were discovered with an orbital visible and near-infrared (VNIR) spectrometer. We will consider the scientific tradeoffs and engineering cost/risks in this instrument selection.

Sample Preparation Station (SPS), based on the Honeybee's Mechanized Sample Handler (MeSH) (Zacny et al., 2014), allows rover-mounted miniature centralized sample preparation. $\mathrm{MeSH}$ crushes small rock cores, then sieves and distributes it in measured aliquots to one or more instrument inlet ports. The system provides a complete core-to-instrument processing solution. It features a fast, efficient, fully automated 8 -axis mechatronic system.

Arm Drill, based on the RANCOR Drill (Zacny et al., 2014) is a rotary percussive coring drill to gather core samples for analysis on Mars. The lightweight drill can capture cores that are $1 \mathrm{~cm}$ in diameter by $6 \mathrm{~cm}$ long. At just over $3 \mathrm{~kg}$, the design provides high performance for an end-of-arm tool in a mass- and power-limited environment. It is built with a robust design for reliable performance: a single actuator drives the bit rotation, creates percussion, and breaks off the core samples for analysis.

Arm Camera, enables high resolution close imaging of sample context before SEM analysis. It would image fine textures that are too coarse for the MVP-SEM but that might be indicative of microbe colonies. The instrument would provide a key step for characterization before SEM nanoimaging and elemental analyses. It is based on the MAHLI cam on the Curiosity rover.

Nav/HazCam uses the Mars 2020 EECAMs design, a $20 \mathrm{M}$ pixel color sensor with a 5120x3840 pixel imaging area. The cameras will also produce color images, with better antiblooming than the MER/MSL designs. To accommodate constraints on downlinked data volume to Earth, the EECAMs can return images at variable resolution. (Maki et al., 2016).

\section{Mars Mapping Drones (MMD) Payload}

MMD are small survey and fetch aerial vehicles that can extend the useful range of the mission. MMD will contain one camera pointing down for mapping and a set pointing outward with 360 degree coverage for navigation and scouting. MMD will have a small sample collection device to collect samples $(<1 \mathrm{~g})$ from remote units and hazardous sites for return and analysis on the rover. While the concept of operations has been developed (ConOps by Skok et al., 2020), this whitepaper focuses on the instrument tradespace that follows.

\subsubsection{Initial Instrument Prioritization}

The mission outlined here is the collection of instruments to fully characterize the proposed science aims. Below, we divide the key instrument systems into four priority levels based on their science return (Figure 4) for cost and risk optimization.

Level 1 would be sufficient to meet the mission objectives, while each additional level would enhance our ability to map, sample and characterize the materials of Mars. 1A) MVP-SEM 
instrument to conduct microscale imaging and compositional analysis. Instrument based on designs by Gaskin (Edmunson, et al., 2016). 1B) Sample Handling subsystem to process natural samples to the size, shape and surface required by the MVP-SEM. Instrument based on Honeybee's Mechanized Sample Handler (MeSH) (Zacny et al., 2014), which can also be replaced by a powder acquisition drill as a single drill and sampling unit (Zacny et al., 2013) 1C) Collection Arm to access geologic samples and deliver them to the Sample Handling subsystem. 1D) Navigation Cameras will allow for visual sample identification. Instrument based on the Mars 2020 EECAM (Maki et al., 2016).

Level 2 would allow enhanced terrain characterization and sample identification. 2A) High-Resolution, multispectral cameras would allow for terrain mapping and contextual information to better characterize the study site and sample context. 2B) Spectrometers would allow mineral mapping and sample targeting. 2C) Arm Imager would allow for high-resolution images of the surface to guide sample selection.

Level 3 would allow greater sample access and control. 3A) Arm Drill would sample at shallow depths and with fine control. Instrument based on HoneyBee RANCOR drill (Zacny et al., 2014). 3B) Mars Mapping Drone (MMD) would provide local mapping, sample reconnaissance and remote sample collection. Instrument based on the JPL Mars Helicopter Scout (Balaram et al., 2018) with the addition of small sample collection capability. 3C) Mars Surface Probe (MSP) would impact the surface before the SPRING landing. Instrument based on the Moon Impact Probe (Goswami \& Annadurai, 2009).

Level 4 would provide enhanced capabilities on the MSP. 4A) Probe Camera would allow for streaming video during the impact flight to improve targeting and provide information to help the main mission landing process. 4B) EDL Platform Spectrometers would allow the study of the dust plume from impact to measure the bulk composition of the near surface.

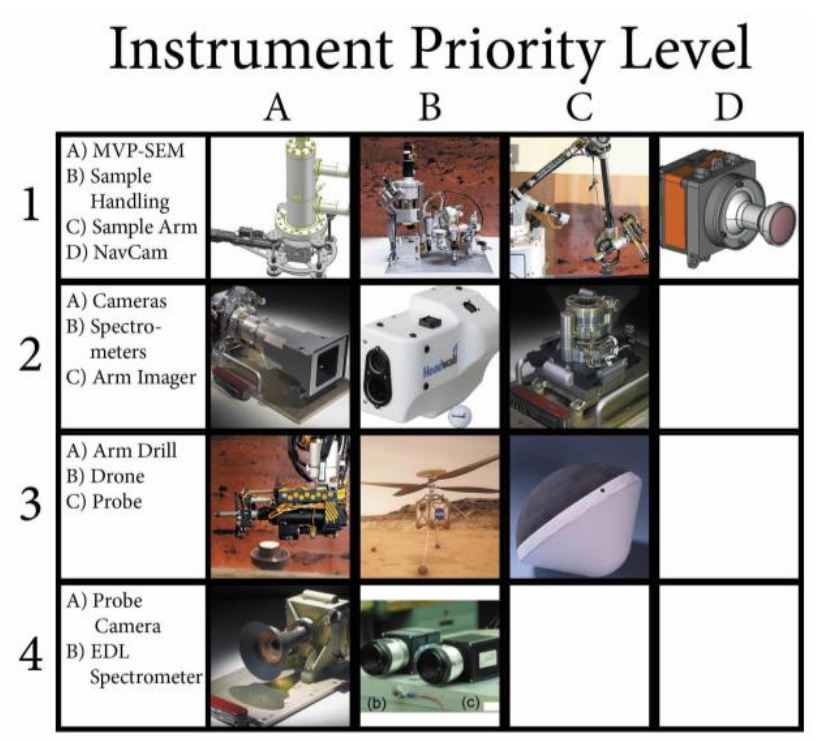

Figure 4: SPRING Mission Instrument Priority Level. Priority 1 would be able to accomplish a complete mission and each additional level (2-4) would enhance the science return. The concept study would develop the engineering and cost constraints to this plan.

\subsubsection{Alternative Target Bodies}

SPRING exemplifies a martian application. However, many of the technologies can be applied to other bodies of interest for the decadal survey. In particular, the MVP-SEM instrument, probe lander and network mapping concept could be modified for use on other planetary bodies. Those include operating on the Moon (or Lunar Gateway), asteroids, comets, Io and Europa, among others. Appropriate modifications of

the SPRING concept would need to be made to account for the different operating conditions of each target.

\subsubsection{Cost Assessment}

The total rough order of magnitude cost for the baseline SPRING Mission is \$950M (FY19) based on previous Mars surface references missions. 
3. References

Balaram et al. (2018) 10.2514/6.2018-0023 - Brasier et al. (2010) 10.1007/978-90-481-8643 3_15

- Cady \& Farmer (1996) 10.1002/9780470514986.ch9 - Campbell et al. (2015) 10.1089/ast.2015.1307 - Chaves Torres et al. (2019) 10.1111/gbi.12340 - Dartnell et al. (2007) 10.1029/2006GL027494 Edmunson, et al. (2016) 47th LPSC Abstract \#2301 - Gibson et al. (2014) 10.1016/j.orggeochem.2014.02.004 - Goswami \& Annadurai (2009) https://www.jstor.org/stable/24105456 - Guido et al. (2010) 10.1144/0016-76492009-109 - Jones et al. (2000) 10.1669/0883-1351(2000)015 - Kaur et al. (2015) 10.1007/s00792-014-0719-9 Kaur et al. (2011) 10.1089/ast.2010.0540 - Kaur et al. (2008) 10.1016/j.orggeochem.2008.04.016 - Konhauser et al. (2001) 10.1046/j.1365-3091.2001.00372.x - Maki et al. (2016) 3rd Int. Workshop on Instrumentation for Plan. Missions Abstract \#4132 - McCollom et al. (2016) Biosig. Preservation and Detection in Mars Analog Enviro. Abstract \#2006 - Munoz-Saez et al. (2016) 10.1016/j.jvolgeores.2016.06.026 - NASEM (2017) 10.17226/24843 - NRC (2011) 10.17226/13117 - Pancost et al. (2005) 10.1111/j.1462-2920.2004.00686.x - Pancost et al. (2006) 10.1111/j.1472-4669.2006.00069.x - Renaut et al. (1998) 10.1046/j.1365-3091.1998.00194.x Ruff et al. (2011) 10.1029/2010JE003767 - Ruff \& Farmer (2016) 10.1038/ncomms13554 - Skok et al. (2010) 10.1038/ngeo990 - Skok et al. (2020) 10.17632/dy37h7fxzb.1 - Squyres et al. (2008) 10.1126/science.1155429 - Smrekar et al. (1999) 10.1029/1999JE001073 - Tarhan et al. (2016) 10.1130/G38542.1 - Teece et al. (2019) Biomolecules from fossilized hot-spring sinters: implications for the search for life on Mars. Astrobiology, submitted - Walter (1996) Ancient hydrothermal ecosystems on earth: a new palaeobiological frontier, in: Ciba Foundation Symposium - Walter \& Des Marais (1993) 10.1006/icar.1993.1011 - Westall et al. (2015) 10.1130/G36646.1 - Westall et al. (2015) 10.1089/ast.2015.1374 - Wolf et al. (2011) 10.1109/AERO.2011.5747243 Williams et al. (2019) Fatty Acid Preservation in Active, Inactive, and Relict Siliceous Sinter Hot Spring Deposits in Iceland, with Implications for Organics Detection on Mars. Astrobiology, submitted - Williams et al. (2020) 51 st LPSC Abstract \#2255 Zacny et al. (2014) 10.1109/AERO.2014.6836211 